\title{
A Review of Tuberculosis in Ndola District of Zambia
}

\author{
Ngula Monde1,2, Musso Munyeme², Sydney Malama ${ }^{3 *}$ \\ ${ }^{1}$ Department of Biomedical Sciences, Tropical Diseases Research Center, Ndola, Zambia \\ ${ }^{2}$ Department of Disease control, School of Veterinary Medicine, University of Zambia, Lusaka, Zambia \\ ${ }^{3}$ Health Promotions Unit, Institute of Economic and Social Research, University of Zambia, Lusaka, Zambia \\ Email: "sydneymalama1971@gmail.com
}

Received 20 January 2016; accepted 5 March 2016; published 8 March 2016

Copyright (C) 2016 by authors and Scientific Research Publishing Inc.

This work is licensed under the Creative Commons Attribution-NonCommercial International License (CC

BY-NC).

http://creativecommons.org/licenses/by-nc/4.0/

(c) (i) (2) Open Access

\section{Abstract}

Background: Tuberculosis (TB) remains the most frequent and important infectious disease causing morbidity and mortality globally. The World Health Organization estimates the incidence of all forms of TB in Zambia at 444/100,000. Tuberculosis case notification rates have increased eightfold over the past two decades and this is largely due to the concurrent Human Immunodeficiency Virus epidemic. The cornerstone of Tuberculosis control is early case detection and treatment which is promoted by Direct Observed Treatment-Short course strategy. This paper reviews the available information in English on TB situation in Ndola district of Zambia with the purpose of assessing successes recorded over the reviewed period. Results: This review has noted a reduction in the number of new cases of TB recorded, improved diagnosis and treatment success of TB in Ndola district over the reviewed period. Conclusion: This review has observed a reduction in the number of new cases of TB recorded in Ndola district over a period of ten years due to the strategies put up by the National TB Program as well as the effective implementation of the strategies by the District Medical team. These strategies included the Directly Observed Therapy Short course (DOTS).

\section{Keywords}

Ndola, Tuberculosis, Zambia

\section{Introduction}

Worldwide, Tuberculosis (TB) remains the most frequent and important infectious disease causing morbidity

"Corresponding author. 
and mortality. Tuberculosis is caused by members of the Mycobacterium tuberculosis complex (MTC), which includes Mycobacterium tuberculosis, Mycobacterium bovis, Mycobacterium africanum, Mycobacterium caprae, Mycobacterium microti, Mycobacterium pinnipedii and Mycobacterium canettii [1] [2]. Approximately one third of the world's population is infected with bacteria belonging to the MTC complex, with Sub-Saharan Africa having the highest annual incidence since the advent of HIV and AIDS [3] [4].

Zambia with a population of 13 million people [5], is an example of a country highly affected by TB (Ayles et al., 2009). Despite a long-running National Tuberculosis and Leprosy Program (NTLP), Zambia has seen a rapid increase in TB cases, especially after 1983, synchronous with the beginning of the HIV era in Zambia [6]. The World Health Organization estimates the prevalence of all forms of TB in Zambia at 444/100,000 and ranks Zambia as ninth in the world [7]. High risk of infection is particularly seen in specific sub-groups such as HIV infected persons, pregnant women, children and prisoners [8]. The HIV prevalence of $14.3 \%$ country wide and $22 \%$ in the capital Lusaka, continues to fuel the TB epidemic with WHO estimating that $70 \%$ of Zambian TB patients are infected with HIV [9]; however, this varies from the different provinces ranging from about $50 \%$ in North-Western Province to more than $80 \%$ in Lusaka province [10]. Other factors which are exacerbating the TB burden in Zambia include high poverty levels, poor housing [5], limited TB control strategies in congregate settings and challenges of TB diagnosis in pediatric patients [11]. The disease burden varies among provinces, with highest notification rates being reported from provinces along the line of rail: Lusaka (28\% in 2004) and Copperbelt (28\% in 2004) followed by Southern Province (10\%) and low rates (5\% to 6\%) from other provinces [12]. The rapid increase of tuberculosis in Zambia from 1985 to 2004 (Figure 1) was mainly attributed to the HIV epidemic, but other factors like population growth, urban overcrowding and improved case detection could have also contributed [13] Zambia has made good progress in TB control since 2007 with the estimated prevalence rates showing a downward trend and having very good treatment outcomes in new cases and retreatment cases regardless of the HIV status of the patients [10].

Mycobacterium tuberculosis is usually transmitted to humans by inhalation of aerosol droplet nuclei containing tubercle bacilli which are expelled by an individual with active pulmonary TB [7]. Although M. tuberculosis infection is the most common cause of human tuberculosis, unknown proportion of cases are due to M. tuberculosis; data on the prevalence of human disease due to M. bovis in Zambia are limited; due to technical problems posed by identification of this species, such as trained personnel and laboratory facilities, only smear microscopy is performed on sputum samples to identify AFB [14].

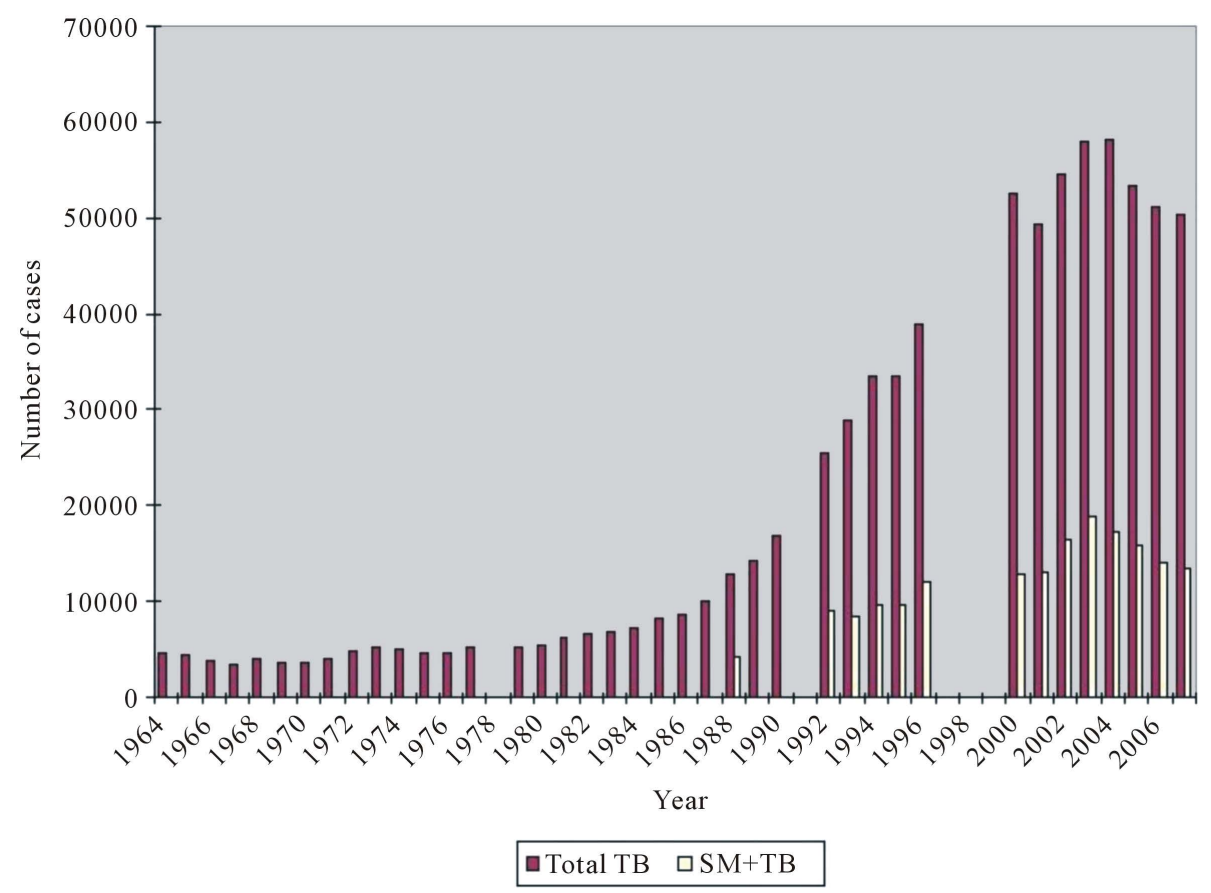

Figure 1. Notifications of tuberculosis all forms and new smear positive cases in Zambia, 1964-2007. Source: NTLP manual, 2014. 


\section{Diagnosis of Tuberculosis in Zambia}

The hallmark of TB control is early case detection and treatment which is promoted by Direct Observed Treatment-Short course (DOTS) strategy [6] [15]. While WHO target detection rate is $75 \%$ of new Smear positive cases and cure rates of $85 \%$, Zambia's performance is only at $49 \%$ and $75 \%$ respectively [7]. The WHO estimates that Zambia has a case detection rate of approximately $80 \%$ [7], meaning that approximately 12,000 TB cases go undiagnosed each year. The public and semi-public sectors notify $90 \%$ of cases; the remainder is from a limited number of private sector facilities that collaborate with the NTLP. Case notification data from Zambia indicate that rates of TB infection and disease have increased eightfold over the past two decades, largely due to the concurrent HIV epidemic [16] [17].

In Zambia, TB is mainly diagnosed by microscopy, using Ziehl-Neelsen (ZN) stains [18]; culture and DST have also been performed since the late 1990s [19]. The ZN method is rapid and inexpensive and highly specific. However, the main limitation of the method is its low sensitivity, with a detection limit of between 5000 10,000 bacilli/ml of sample, particularly in HIV co-infected patients [20]. With these limitations of ZN technique Zambia has rolled out the use of LED fluorescent microscopy in most of the TB diagnostic centers in the country.

The laboratory gold standard for diagnosing TB is mycobacterial culture [21]. Mycobacterial culture is much more sensitive than microscopy and allows the recovery of the bacteria for other studies, such as drug susceptibility testing and genotyping [21]. In Zambia, due to limited infrastructure in most health facilities, culture is only performed in the three TB reference laboratories situated in Lusaka and Ndola districts and a few selected private institutions.

Drug susceptibility testing to $1^{\text {st }}$ line drugs is mainly performed using convertional methods such as the proportional method using Lowestein-Jensen (L-J) and liquid medium based methods such as Mycobacterial Growth Indicator Tube (MGIT) system 960 (BD diagnostics, USA). Culture and DST is recommended to be performed only on samples from all patients enrolled on treatment as retreatment cases; those who fail to respond to treatment and all those who had interrupted treatment. The drugs routinely tested for are rifampicin, isoniazid, ethambutol and streptomycin [22].

In recent years, a number of alternative diagnostic tests that use immunological and molecular methods have been developed. Many molecular methods have been developed for direct detection, species identification and drug susceptibility testing of mycobacteria [23]. One of the new Rapid molecular test that has the potential to substantially improve and accelerate the diagnosis of TB and drug resistance TB is the GeneXpert MTB/RIF diagnostic test. The Xpert MTB/RIF system is a recently developed TB-specific application, designed to detect M. tuberculosis as well as rifampicin resistance-conferring mutations directly from sputum [24]. The recent WHO endorsement of Xpert MTB/RIF is a good development; however, the technology in Zambia is being implemented in phases and only in selected health facilities. This is because financing the implementation of such a molecular test is a major undertaking [19] [25]. Another molecular method being used in Zambia is the Line Probe Assay (Hain Lifescience, Germany). The Line Probe Assay ( $1^{\text {st }}$ line) is a DNA strip test that allows simultaneous molecular identification of tuberculosis and the most common genetic mutations causing reistance to rifampicin and isoniazid (FIND-line probe assay, 2015). However, this method is being used in the Reference laboratories where culture, biosafety and PCR facilities exist. Although molecular methods are rapid and results are obtainable within a limited period of time, these techniques need more advanced and sophisticated infrastructure and their introduction in the country like Zambia on routine bases requires careful planning and additional human and financial resources [13].

\section{Treatment of TB in Zambia}

The standard treatment regimen for new case has an initial phase lasting 2 months using for 4 drugs, isoniazid, rifampicin, pyrazinamide, and ethambutol. In the continuation phase lasting 4 months, 2 drugs, isoniazid and rifampicin are used [19] [26]. WHO recommends that national TB control programmes should ensure that supervision and support are provided for all TB patients in order to achieve completion of the full course of therapy [27]. Treatment success rate for new TB smear positive cases has greatly improved from $77 \%$ in 2002 cohort to 88\% for the 2012 cohort in Zambia. A study done by Kapata et al., where data on the trends of TB burden over the past two decades was reviewed, indicated that the cure rates almost doubled in the last decade from $47 \%$ in 2000 to $82 \%$ in 2009. The treatment success rate also increased from $66 \%$ in 2000 to $86 \%$ in 2009 [18]. 


\section{TB Situation in Ndola District}

Ndola district is located on the Copperbelt of Zambia and is the third largest city in Zambia with a population of 455, 194 [5]. Ndola lies between latitudes $120^{\circ}$ and $160^{\circ}$ South of the Equator and between $250^{\circ}$ and $30^{\circ}$ east (Figure 2). Ndola district has 31 health facilities: 28 primary health centers or clinics, two referral hospitals and one infectious disease reference laboratory. Most of these health centres provide TB diagnosis and treatment.

Sputum smear microscopy still remains the cornerstone for diagnosis of TB in Ndola District. A study done by Mulenga et al., 2010 showed that the majority of MTBC isolates in Ndola belongs to the SAF1 family with a high clustering rate indicating high recent transmission of TB [6]. This emphasizes on the importance of early diagnosis and timely treatment. Differentiation within the Mycobacterium tuberculosis complex is necessary for epidemiological purposes and some individual patient treatment [28]. Since TB in Ndola is mostly diagnosed by sputum smear microscopy (SSM) which cannot differentiate members of the complex. The epidemiology of TB remains largely unknown, hence, posing challenges in TB control strategies. Routine acid-fast bacilli (AFB) culture and identification of isolates are not performed at each TB diagnostic centre due to lack of well-equipped laboratory facilities [18]. However, the district has one regional TB laboratory which performs culture only follow up cases such as treatment failures, defaulters etc.

\section{TB Intervention Strategies in Ndola District}

Data from Ndola DHMT show that there has been a downward trend in the number of new cases of TB from 2002 to 2014 in Ndola urban district (Figure 3). These could be due to improvement in TB intervention strategies such as Directly Observed Therapy Short course (DOTS) implementation. DOTS remain at the heart of the strategy to stop TB. DOTS consists of 5 main technical elements; political commitment, case detection through quality assured bacteriology, standardized treatment with supervision and patient support, an effective drug supply and management system and a monitoring and evaluation system that incorporates impact measurement [15]. Zambia adopted the WHO-recommended DOTS strategy as its primary approach for tuberculosis (TB) control in 1993 and has since achieved 100\% coverage. Ndola district is one of the earliest to have implemented DOTS in Zambia [15]. In an effort to improve case management, Ndola District has benefited from the use of community volunteers and treatment supporters in their TB programme to assist in ensuring patients complete treatment. DHMT data for Ndola district for 2014 indicate a relatively high treatment success rate of 91\% [29]. High treatment success rates may contribute to deceased notification rates and may indicate decreased TB transmission in the general public [22]. There has also been an improvement in diagnosis of TB, meaning that all the TB suspects are being confirmed as cases. Some years back, most cases of pulmonary tuberculosis were

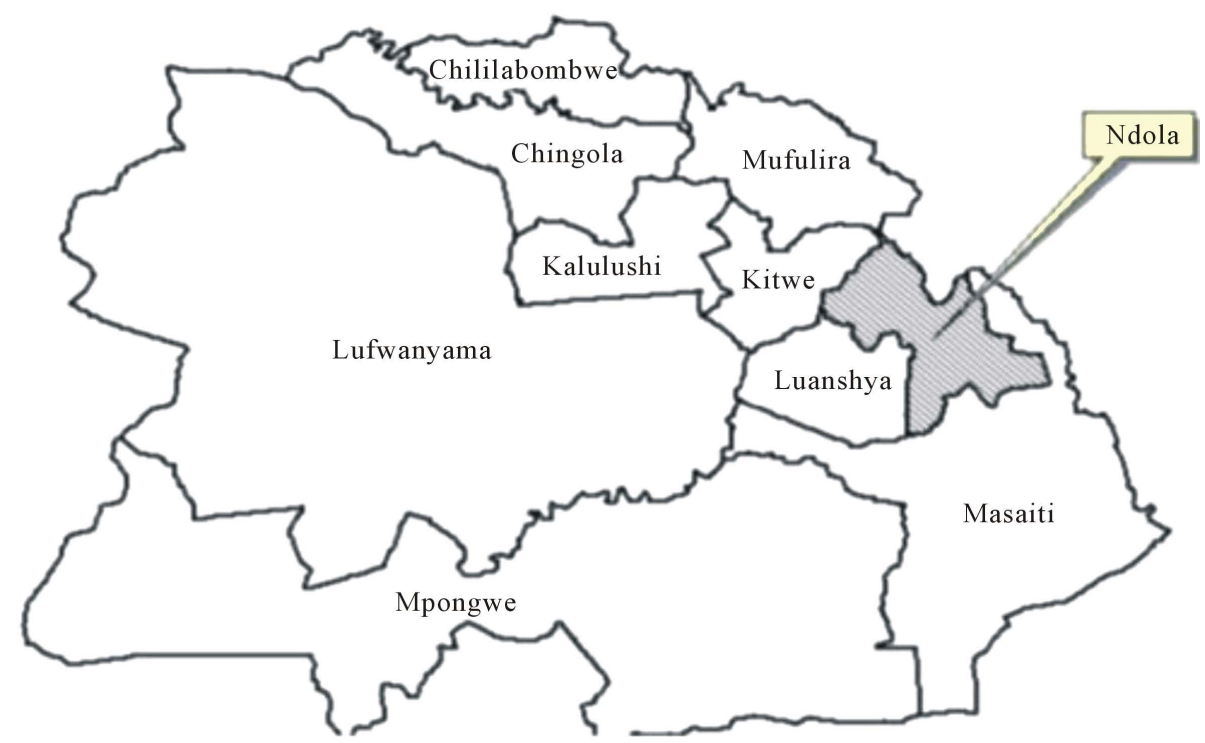

Figure 2. Map depicting Ndola district of Zambia. Source: City of Ndola-District situation analysis, 2005. 


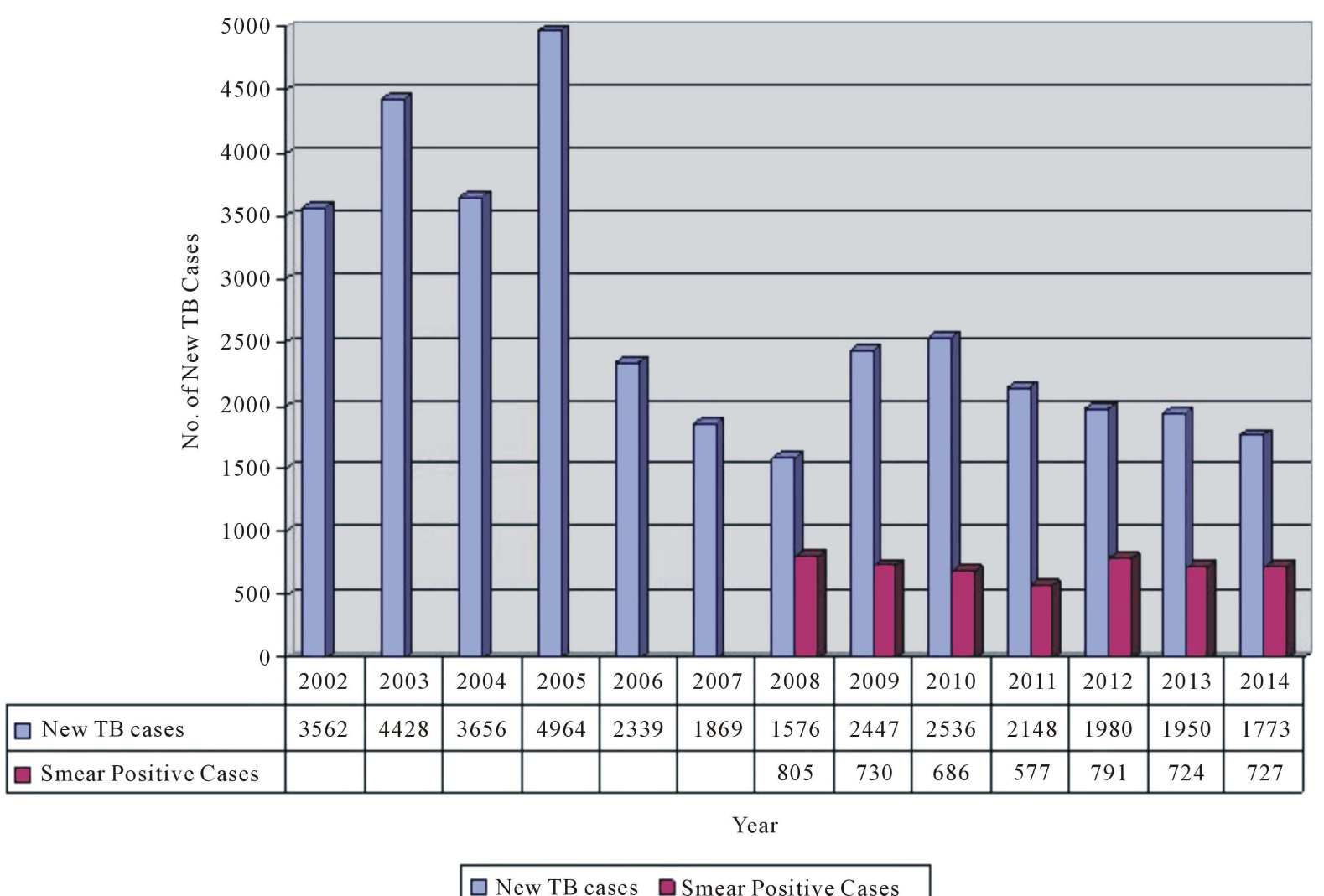

Figure 3. Number of New TB cases and Smear positive cases from 2002 to 2014 Ndola District. Source: Ndola DHMT data, 2002-2014.

diagnosed through clinical diagnoses and light microscopy which has proved to be less sensitive. This means that in some cases, patients were diagnosed with TB disease on the basis of their signs and symptoms or using chest X-ray, even if their specimen does not contain M. tuberculosis resulting in over diagnosis of TB. Most of the diagnostic centres in Ndola district are performing smear microscopy using the more sensitive and WHO recommended LED fluorescence Microscopy. The NTLP has also rolled out the use of GeneXpert technology in selected health facilities with the help of partners such as TB Care II. There has also been an effective screening of TB contacts. It is recommended that household contacts be screened for symptoms of disease and Isoniazid preventive therapy (i.e. daily Isoniazid for at least 6 months) offered to children aged less than 5 years along with all HIV-infected children [30]. This is to reduce the risk of infection.

There has also been an effective supply of anti TB drugs in the district. The district has not experienced any shortages of these drugs for the past three to four years, this could also contribute to the experienced downward trend in new TB cases [31].

The other reasons for the downward trend could be weaknesses in the routine TB surveillance. The routine surveillance data may have gaps due to recording bias or weaknesses. This observed downward trend also entail that the National TB control programme is performing very well in reducing the burden of TB in the district. This is in terms of provision of quality diagnostic and treatment services for TB cases at every level of health delivery. In order to provide quality TB services, the NTLP has developed guidelines and standards to explain the different steps involved in TB management, including the National TB Guidelines and the TB Manual. Separate guidelines also exist for adult TB, MDR TB, TB/HIV, TB infection control, and procurement and supply management. Standards on diagnosis and treatment and laboratory manuals have also been developed; as well as forms, formats, and a standardized system for record keeping; and systems for supervision, monitoring and evaluation [29]. Ndola district has also implemented the TB infection control demonstration project at most of its diagnostic centres through the Ministry of Health (MOH) and the Ministry of Community Development Mother and Child Health (MCDMCH) with the support of TB CARE I and TB CARE II partners. The goal of 
the project was to provide safe work practices in order to reduce TB transmission among people living with HIV (PLHIV) and Health Care Workers (HCWs). The implementation process was aimed at strengthening the district healthcare system in implementing TB Infection Control, acknowledging occupational risk for the health care workers acquiring TB infection and developing TB disease [31].

\section{Conclusion}

This review has observed a reduction in the number of new cases of TB recorded in Ndola district over the reviewed period due to the strategies such as DOTS put up by the National TB Program as well as the effective implementation of these strategies by the District Medical team. However, TB is a disease that has always been, and still is associated with poverty, HIV/AIDS and deprivation. Therefore, the eventual eradication of this ancient scourge will surely go hand in hand with the creation of a caring national society and improved living standards of the people.

\section{Acknowledgements}

The authors wish to thank the Ndola District Health Management Team for their cooperation and assistance during data collection.

\section{Recommendations}

- Newer diagnostics technologies for the rapid diagnosis of active TB cases and drug resistance testing such as the GeneXpert MTB/RIF technology needs to be fully funded by the NTLP in order to sustain its implementation in the selected health facilities in Ndola.

- There is also need to improve case detection and treatment of TB in children.

- There is need to ensure that all the necessary materials are provided to all TB diagnostic centers to ensure accurate and prompt diagnosis of TB.

- There is need for studies to be done on non tuberculous Mycobacteria and ascertain its prevalence in humans in Ndola district.

\section{Competing Interests}

The authors declare that they have no competing interests.

\section{Authors' Contributions}

NM contributed to reviewing literature and drafting of the manuscript, MM contributed to the writing of the manuscript, SM contributed to the conception, reviewing literature and editing of the manuscript. All authors read and approved the final manuscript.

\section{References}

[1] Botha, L., van Pittius, N.C. and Helden, P.D. (2013) Mycobacteria and Disease in Southern Africa. Transboundary and Emerging Diseases, 60, 147-156. http://dx.doi.org/10.1111/tbed.12159

[2] Brosch, R., Gordon, S.V., Marmiesse, M., Brodin, P., Buchrieser, C., Eiglmeier, K., et al. (2002) A New Evolutionary Scenario for the Mycobacterium tuberculosis Complex. Proceedings of the National Academy of Sciences of the United States of America, 99, 3684-3689. http://dx.doi.org/10.1073/pnas.052548299

[3] Daborn, C.J. and Grange, J.M. (1993) HIV/AIDS and Its Implications for the Control of Animal Tuberculosis. British Veterinary Journal, 149, 405-417. http://dx.doi.org/10.1016/S0007-1935(05)80107-1

[4] Malama, S., Muma, J.B. and Godfroid, J. (2013) A Review of Tuberculosis at the Wildlife-Livestock-Human Interface in Zambia. Infectious Diseases of Poverty, 2, 13. http://dx.doi.org/10.1186/2049-9957-2-13

[5] CSO (2011) Zambia 2010 Census of Population and Housing: Central Statistical Office (CSO).

[6] Mulenga, C., Shamputa, I.C., Mwakazanga, D., Kapata, N., Portaels, F. and Rigouts, L. (2010) Diversity of Mycobacterium tuberculosis Genotypes Circulating in Ndola, Zambia. BMC Infectious Diseases, 10, 177. http://dx.doi.org/10.1186/1471-2334-10-177

[7] WHO (2013) Global Tuberculosis Report 2013: ECD-WHO/Europe Joint Surveillance. 
[8] (2011) Centre for Infectious Diseases Research in Zambia Annual Report.

[9] Ayles, H., Schaap, A., Nota, A., Sismanidis, C., Tembwe, R., De Haas, P., et al. (2009) Prevalence of Tuberculosis, HIV and Respiratory Symptoms in Two Zambian Communities: Implications for Tuberculosis Control in the Era of HIV. PLOS ONE, 4, e5602. http://dx.doi.org/10.1371/journal.pone.0005602

[10] Kapata, N., Chanda-Kapata, P., O’Grady, J., Bates, M., Mwaba, P., Janssen, S., et al. (2012) Trends in Childhood Tuberculosis in Zambia: A Situation Analysis. Journal of Tropical Pediatrics, fms065.

[11] Zumla, A., Squire, S.B., Chintu, C. and Grange, J.M. (1999) The Tuberculosis Pandemic: Implications for Health in the Tropics. Transactions of the Royal Society of Tropical Medicine and Hygiene, 93, 113-117. http://dx.doi.org/10.1016/S0035-9203(99)90278-X

[12] (2004) Central Board of Health, Zambia Annual Report.

[13] (2014) Zambia National TB Manual.

[14] Malama, S., Muma, J.B., Olea-Popelka, F. and Mbulo, G. (2013) Isolation of Mycobacterium bovis from Human Sputum in Zambia: Public Health and Diagnostic Significance. Journal of Infectious Diseases and Therapeutics, 1, 114.

[15] Mulenga, C., Mwakazanga, D., Vereecken, K., Khondowe, S., Kapata, N., Shamputa, I.C., et al. (2010) Management of Pulmonary Tuberculosis Patients in an Urban Setting in Zambia: A Patient's Perspective. BMC Public Health, 10, 756. http://dx.doi.org/10.1186/1471-2458-10-756

[16] Piot, P., Bartos, M., Larson, H., Zewdie, D. and Mane, P. (2008) Coming to Terms with Complexity: A Call to Action for HIV Prevention. The Lancet, 372, 845-859. http://dx.doi.org/10.1016/S0140-6736(08)60888-0

[17] Aspler, A., Menzies, D., Oxlade, O., Banda, J., Mwenge, L., Godfrey-Faussett, P., et al. (2008) Cost of Tuberculosis Diagnosis and Treatment from the Patient Perspective in Lusaka, Zambia. The International Journal of Tuberculosis and Lung Disease, 12, 928-935.

[18] Kapata, N., Chanda-Kapata, P., Grady, J., Schwank, S., Bates, M., Mukonka, V., et al. (2011) Trends of Zambia Tuberculosis Burden over the Past Two Decades. Tropical Medicine \& International Health, 16, 1404-1409. http://dx.doi.org/10.1111/j.1365-3156.2011.02849.X

[19] O’Grady, J., Maeurer, M., Mwaba, P., Kapata, N., Bates, M., Hoelscher, M., et al. (2011) New and Improved Diagnostics for Detection of Drug-Resistant Pulmonary Tuberculosis. Current Opinion in Pulmonary Medicine, 17, $134-141$. http://dx.doi.org/10.1097/MCP.0b013e3283452346

[20] Albert, H., Manabe, Y., Lukyamuzi, G., Ademun, P., Mukkada, S., Nyesiga, B., et al. (2010) Performance of Three LED-Based Fluorescence Microscopy Systems for Detection of Tuberculosis in Uganda. PLoS ONE, 5, e15206. http://dx.doi.org/10.1371/journal.pone.0015206

[21] Muwonge, A., Malama, S., Bronsvoort, B.M., Biffa, D., Ssengooba, W., Skjerve, E. (2014) A Comparison of Tools Used for Tuberculosis Diagnosis in Resource-Limited Settings: A Case Study at Mubende Referral Hospital, Uganda. PLoS ONE, 9, e100720. http://dx.doi.org/10.1371/journal.pone.0100720

[22] Henostroza, G., Topp, S.M., Hatwiinda, S., Maggard, K.R., Phiri, W., Harris, J.B., et al. (2013) The High Burden of Tuberculosis (TB) and Human Immunodeficiency Virus (HIV) in a Large Zambian Prison: A Public Health Alert. PLoS ONE, 8, e67338. http://dx.doi.org/10.1371/journal.pone.0067338

[23] Malama, S. (2013) Isolation and Molecular Characterisation of Mycobacterium Tuberculosis Complex at the HumanAnimal Interface of Namwala District, Zambia. PhD Thesis, Norwegian University of Life Sciences, Oslo.

[24] Hillemann, D., Rüsch-Gerdes, S., Boehme, C. and Richter, E. (2011) Rapid Molecular Detection of Extrapulmonary Tuberculosis by the Automated GeneXpert MTB/RIF System. Journal of Clinical Microbiology, 49, 1202-1205. http://dx.doi.org/10.1128/JCM.02268-10

[25] Lawn, S.D., Mwaba, P., Bates, M., Piatek, A., Alexander, H., Marais, B.J., et al. (2013) Advances in Tuberculosis Diagnostics: The Xpert MTB/RIF Assay and Future Prospects for a Point-of-Care Test. The Lancet Infectious Diseases, 13, 349-361. http://dx.doi.org/10.1016/S1473-3099(13)70008-2

[26] (2009) National TB Management Guidelines.

[27] World Health Organisation (2009) Global Tuberculosis Control WHO Report. WHO/HTM/TB/2009.411, Geneva.

[28] Muwonge, A., Malama, S., Johansen, T.B., Kankya, C., Biffa, D., Ssengooba, W., et al. (2013) Molecular Epidemiology, Drug Susceptibility and Economic Aspects of Tuberculosis in Mubende District, Uganda. PLoS ONE, 8, e64745. http://dx.doi.org/10.1371/journal.pone.0064745

[29] (2014) TB Care II—Ndola District TBIC Demonstration Project Final Report.

[30] O’Grady, J., Maeurer, M., Mwaba, P., Kapata, N., Bates, M., Hoelscher, M., et al. (2011) New and Improved Diagnostics for Detection of Drug-Resistant Pulmonary Tuberculosis. Current Opinion in Pulmonary Medicine, 17, $134-141$. http://dx.doi.org/10.1097/MCP.0b013e3283452346 
[31] (2013) TB Care II-Quality Improvement in Tb Services. Assessment of Provider Adherance to TB Evidence Based Standards and Guidelines in Zambia.

\section{Abbreviations}

$\begin{array}{ll}\text { CSO } & \text { Central Statistics Office } \\ \text { DHMT } & \text { Ndola Health Management Team } \\ \text { DOTS } & \text { Direct Observed Treatment Short course } \\ \text { LED FM } & \text { Light Emitting Diode Fluorescence Microscopy } \\ \text { MCDMCH } & \text { Ministry of Community Development, Mother and Child Health } \\ \text { MDR } & \text { Multidrug Resistance } \\ \text { MOH } & \text { Ministry of Health } \\ \text { NTLP } & \text { National Tuberculosis and Leprosy control Programme } \\ \text { PLHIV } & \text { People Living with HIV } \\ \text { WHO } & \text { World Health Organization } \\ \text { ZDHS } & \text { Zambia Demographic Health Survey }\end{array}$

